\title{
BMJ Open Updating EuroSCORE by including emotional, behavioural, social and functional factors to the risk assessment of patients undergoing cardiac surgery: a study protocol
}

\author{
Pernille Fevejle Cromhout, ${ }^{1} 1$ Selina Kikkenborg Berg, ${ }^{2,3}$ Philip Moons, ${ }^{4,5}$ \\ Sune Damgaard, ${ }^{6}$ Samer Nashef, ${ }^{7}$ Lau Caspar Thygesen ${ }^{3}$
}

To cite: Cromhout PF, Berg SK, Moons $\mathrm{P}$, et al. Updating EurOSCORE by including emotional, behavioural, social and functional factors to the risk assessment of patients undergoing cardiac surgery: a study protocol. BMJ Open 2019;9:e026745. doi:10.1136/ bmjopen-2018-026745

- Prepublication history and additional material for this paper are available online. To view please visit the journal (http:// dx.doi.org/10.1136/bmjopen2018-026745).

Received 17 September 2018 Revised 24 May 2019 Accepted 30 May 2019

D Check for updates

(c) Author(s) (or their employer(s)) 2019. Re-use permitted under CC BY-NC. No commercial re-use. See rights and permissions. Published by BMJ.

For numbered affiliations see end of article.

\section{Correspondence to} Pernille Fevejle Cromhout; pernille.fevejle.cromhout@ regionh.dk

\section{ABSTRACT}

Introduction Conventional risk assessment in cardiac surgery focus on medical and physiological factors and have been developed to predict mortality. Other relevant risk factors associated with increased risk of poor outcomes are not included. Adding nonmedical variables as potential prognostic factors to risk assessments direct attention away from specific diagnoses towards a more holistic view of the patients and their predicament. The aim of this paper is to describe the method and analysis plan for the development and validation of a prognostic screening tool as a supplement to the European System for Cardiac Operative Risk Evaluation (EuroSCORE) to predict mortality, readmissions and prolonged length of admission in patients within 90 days after cardiac surgery, as individual outcomes.

Methods and analysis The development of a prognostic screening tool with inclusion of emotional, behavioural, social and functional factors complementing risk assessment by EuroSCORE will adopt the methods recommended by the PROGnosis RESearch Strategy Group and report using the Transparent Reporting of a multivariable prediction model for Individual Prognosis Or Diagnosis statement. In the development stage, we will use data derived from three datasets comprising 1143, 3347 and 982 patients for a prospective cohort study of patients undergoing cardiac surgery, respectively. We will construct logistic regression models to predict mortality, prolonged length of admission and 90-day readmissions. In the validation stage, we will use data from a separate sample of 333 patients planned to undergo cardiac surgery to assess the performance of the developed prognostic model. We will produce validation plots showing the overall performance, area under the curve statistic for discrimination and the calibration slope and intercept. Ethics and dissemination The study will follow the requirements from the Ethical Committee System ensuring voluntary participation in accordance with the Helsinki declarations. Data will be filed in accordance with the requirements of the Danish Data Protection Agency.
Strengths and limitations of this study

- Follows the PROGnosis RESearch Strategy framework, and Transparent Reporting of a multivariable prediction model for Individual Prognosis Or Diagnosis reporting guidelines for prognostic research.

- Prespecifies statistical analysis plan and informative levels of tool performance to increase transparency of results and of the final reporting.

- Use of national administrative registers.

- Restricted to the use of predictor variables measured in previous datasets.

- Minor differences in the way variables are measured between the development and the validation datasets.

\section{INTRODUCTION}

Today cardiac surgery is being performed on an older, more complex and high-risk population than ever. This calls for tertiary preventive initiatives for preventing poor outcomes such as mortality and readmissions based on prognostic factors. ${ }^{1-6}$ Prognostic factors can be combined to produce prognostic models used to screen patients into risk groups. ${ }^{7}$ Early identification of at-risk patients allows healthcare professionals to make informed decisions based on prognostic profiles and enables patients to understand the possible risks of surgery. Conventional prognostic screening tools focus on medical and physiological factors, but do not contain other risk factors associated with poor outcomes following cardiac surgery. ${ }^{8}$

There are several prognostic screening tools currently available for patients undergoing cardiac surgery. In most of Europe European System for Cardiac Operative Risk Evaluation (EuroSCORE) ${ }^{9}{ }^{10}$ is used in 
Prognostic multivariable modelling study

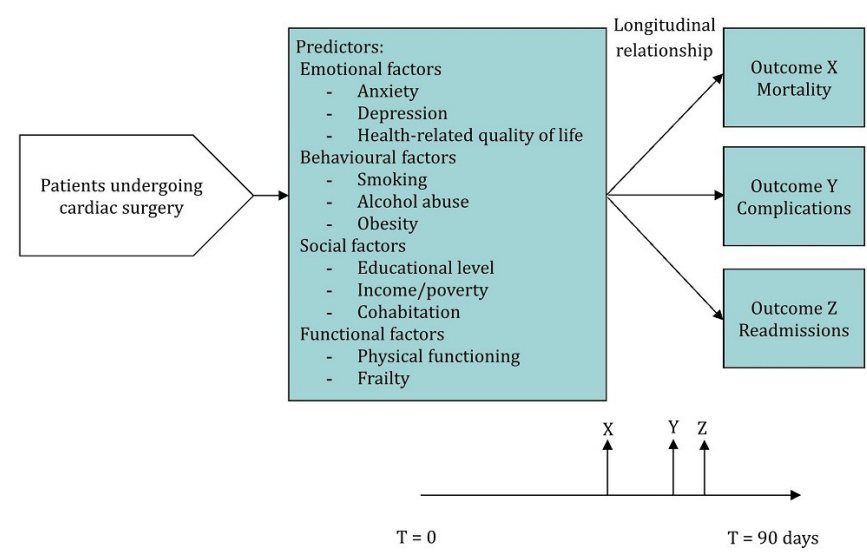

Figure 1 Schematic presentation of prognostic prediction modelling study.

clinical practice. EuroSCORE was developed for in-hospital and 30-day mortality and has been widely used and recommended for clinical use, not only for operative risk prediction but also for the assessment of the quality of cardiac surgery. ${ }^{9-12}$ The total risk is calculated by adding scores from several risk factors (eg, chronic pulmonary disease, serum creatinine and left ventricular dysfunction) resulting in a predicted percentage of surgical mortality. The score with a range of $0-22$ is defined to distinguish low $(<3)$, moderate (3-5) and high-risk groups $(6+) .{ }^{9}{ }^{10}$ However, validation studies have reported that EuroSCORE is inaccurate in predicting mortality rates. $^{13-15}$

Indeed, there is mounting evidence that non-physiological factors, such as emotional, ${ }^{16-18}$ behavioural, ${ }^{19-21}$ social $^{22-27}$ and functional ${ }^{2829}$ factors are predictive of poor outcomes following cardiac surgery. ${ }^{8}$ Adding non-medical variables as potential prognostic factors directs attention away from specific diagnoses towards a more holistic view of the patient and their predicament. The study described in this protocol is planned as the developing and validation of risk stratification models complementing EuroSCORE with emotional, behavioural, social and functional factors to predict mortality, readmissions and prolonged length of admission in patients within 90 days after cardiac surgery.

Most existing prognostic tools have been developed to predict mortality only. Mortality is a reliable and clinically important outcome in cardiac surgery; however, there are other measures and other adverse outcomes that might be worth considering. One of these is readmission, which is an outcome with significant health and economic implications. ${ }^{4}$ Another important process outcome in cardiac surgery is prolonged length of stay in the intensive care unit (ICU) and in hospital. ${ }^{30}$

The aim of this paper is to describe the method and analysis plan for the development and validation of a prognostic screening tool as a supplement to EuroSCORE to predict mortality, readmissions and prolonged length of stay in patients within 90 days after cardiac surgery.

\section{METHODS AND ANALYSIS}

The study is based on data from different data sources allocated to different datasets. Primary outcome is death and secondary outcomes are prolonged length of ICU stay and hospital admission as well as readmissions, all within 90 days from the time of cardiac surgery. Inclusion criteria are age $\geq 18$ years and planned for or having undergone cardiac surgery. Patients will be excluded if data on EuroSCORE are not available. Multiple regression analyses will be performed to investigate the association between emotional, social, functional and behavioural variables and outcomes. In the model development, EuroSCORE is included while each potential predictor will be removed stepwise from the model by using an automated backwards selection procedure. The final model will be prospectively tested to determine its predictive validity.

\section{Study design and study population}

This paper is inspired by the PROGnosis RESearch Strategy (PROGRESS) framework ${ }^{6} \quad 3132$ and will be reported in accordance with the Transparent Reporting of a multivariable prediction model for Individual Prognosis Or Diagnosis (TRIPOD). ${ }^{33}$ This is V.1.2 of the protocol.

Cardiac surgery typically includes standardised procedures especially for coronary artery bypass grafting and

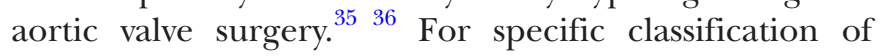
included procedures, see online supplementary table 1.

\section{Development stage}

\section{Development sample}

In the development stage, prognostic factors will be identified and combined to a prognostic model based on emotional, behavioural, social and functional factors in addition to the EuroSCORE to predict mortality, readmissions and prolonged length of admission in patients within 90 days after cardiac surgery. See figure 1 for a schematic presentation of the prognostic prediction modelling. The need for follow-up beyond the traditional 30 days, has previously been advanced since the 30-day mortality only reflects part of early mortality after cardiac surgery. $^{37}$

The development sample consists of three datasets (table 1). Dataset 1 consists of primary data collected from October 2016 to February 2018. Included patients participated in a preoperative survey including all patients $\geq 18$ years planned for cardiac surgery at Copenhagen University hospital, Rigshospitalet $(n=1143)$. Sample size were estimated based an expected incidence of death following cardiac surgery of $3.5 \%$ and including four covariates (see table 2). Information on preoperative symptoms of anxiety, depression and frailty (when aged $\geq 70$ years) are included (see flowchart, figure 2). Dataset 2 consists of existing data from Danish national registers, including 
Table 1 Prespecification of predictor variables with initial df allocation

\begin{tabular}{|c|c|c|c|}
\hline & Variable & df & Codes/values \\
\hline \multirow[t]{3}{*}{$\begin{array}{l}\text { Social } \\
\text { factors }\end{array}$} & Educational level & 2 & $\begin{array}{l}0=\text { =Basic school } \\
1=\text { Upper secondary } \\
\text { or vocational school } \\
2=\text { Higher education }\end{array}$ \\
\hline & $\begin{array}{l}\text { Equivalised } \\
\text { disposable } \\
\text { income }\end{array}$ & 2 & $\begin{array}{l}0=\leq 50 \% \text { median } \\
1=>50 \%- \\
150 \% \text { median } \\
2=\geq 150 \% \text { median }\end{array}$ \\
\hline & $\begin{array}{l}\text { Cohabitation } \\
\text { status }\end{array}$ & 1 & $\begin{array}{l}0=\text { Non-cohabitation } \\
1=\text { Cohabitation }\end{array}$ \\
\hline \multirow[t]{3}{*}{$\begin{array}{l}\text { Functional } \\
\text { factors }\end{array}$} & $\begin{array}{l}\text { Frailty-Gait } \\
\text { speed test }\end{array}$ & 1 & $\begin{array}{l}0=\text { No } \\
1=\text { Yes }\end{array}$ \\
\hline & $\begin{array}{l}\text { SF-12, physical } \\
\text { component scale }\end{array}$ & 1 & $\begin{array}{l}0=\geq \text { mean }-1 S D \\
1=<\text { mean }-1 S D\end{array}$ \\
\hline & $\begin{array}{l}\text { HeartQoL- } \\
\text { physical }\end{array}$ & 1 & $\begin{array}{l}0=\geq \text { median } \\
1=<\text { median }\end{array}$ \\
\hline \multirow[t]{3}{*}{$\begin{array}{l}\text { Behavioural } \\
\text { factors }\end{array}$} & Smoking & 2 & $\begin{array}{l}0=\text { Never } \\
1=\text { Former } \\
2=\text { Current }\end{array}$ \\
\hline & $\begin{array}{l}\text { Alcohol abuse } \\
\text { male: }>21 \text { drinks/ } \\
\text { week } \\
\text { Women: >14 } \\
\text { drinks/week }\end{array}$ & 1 & $\begin{array}{l}0=\text { No } \\
1=\text { Yes }\end{array}$ \\
\hline & Body mass index & 2 & $\begin{array}{l}0=\leq 25 \\
1=26-30 \\
2=>30\end{array}$ \\
\hline \multirow[t]{5}{*}{$\begin{array}{l}\text { Emotional } \\
\text { factors }\end{array}$} & $\begin{array}{l}\text { SF-12, mental } \\
\text { component scale }\end{array}$ & 1 & $\begin{array}{l}0=\geq \text { mean }-1 S D \\
1=<\text { mean }-1 S D\end{array}$ \\
\hline & HADS-anxiety & 2 & $\begin{array}{l}0=<8 \\
1=8-10 \\
2=\geq 11\end{array}$ \\
\hline & $\begin{array}{l}\text { HADS- } \\
\text { depression }\end{array}$ & 2 & $\begin{array}{l}0=<8 \\
1=8-10 \\
2=\geq 11\end{array}$ \\
\hline & HeartQoL-global & 1 & $\begin{array}{l}0=\geq \text { median } \\
1=<\text { median }\end{array}$ \\
\hline & $\begin{array}{l}\text { HeartQoL- } \\
\text { emotional }\end{array}$ & 1 & $\begin{array}{l}0=\geq \text { median } \\
1=<\text { median }\end{array}$ \\
\hline
\end{tabular}

HADS, Hospital Anxiety and Depression Scale; SF-12, 12-Item Short-Form Health Survey.

all patients having undergone cardiac surgery from April 2013 to April 2014 at all four Danish hospitals performing cardiac surgery $(n=3217)$. Information on socioeconomic factors and cohabitation are obtained through register linkage (see flow-chart, figure 3). Dataset 3 is a subsample of dataset 2 and includes all patients who participated in the DenHeart study ( $\mathrm{n}=982)$, a national survey including all cardiac diagnostic groups. ${ }^{38}$ Information on self-reported health and quality of life were collected on discharge through validated questionnaires (see flowchart, figure 4).
The three datasets will be linked with national administrative registers for information on mortality, prolonged length of stay and readmissions.

\section{Data sources}

Two population-based, administrative registers were used in this study: (1) the Danish National Patient Register (DNPR), which has registered information on somatic conditions leading to hospitalisation since 1978 and, from 1995, also outpatient visits ${ }^{39}$ and (2) the Danish Civil Registration System (CRS). All Danish residents have since 1968 been assigned a unique personal identification number and have been registered in the CRS by date of birth, sex, marital status, migration and vital status. ${ }^{40}$ The personal identification number allows accurate linkage with national administrative registers. These registers have previously been well validated. ${ }^{4041}$ The clinical databases utilised for information on EuroSCORE and ICU admission cover all regions of Denmark. For Eastern Denmark, EuroSCORE II was not implemented for the time period covering datasets 2 and 3. According to the yearly reports data completeness for risk profile is $100 \%$ and $\geq 98 \%$ for ICU admission. ${ }^{42}$

The DenHeart study was a cross-sectional survey conducted April 2013 to April 2014, consisting of six validated questionnaires and several ancillary questions, with a total of 80 questions. The survey included nine questions about health and health behaviour from the Danish Health and Morbidity Survey and the Danish National Health Survey ${ }^{43}$ and one question regarding medication adherence. All cardiac patients were asked to fill out the questionnaire at hospital discharge to evaluate patient-reported outcomes. ${ }^{38}$ The survey data were combined with data from the registers by matching with a hospital discharge from the DNPR.

\section{Outcomes}

One primary and three secondary outcomes are included in this study. Each of the outcomes will be evaluated individually.

\section{Primary outcome}

The primary outcome is mortality up to 90 days after cardiac surgery.

\section{Secondary outcomes}

\section{Readmissions}

Nearly $20 \%$ of patients undergoing cardiac surgery require acute readmission after surgery. ${ }^{4}$ That makes readmissions following cardiac surgery an outcome with significant health and economic implications. Readmission will be included as a dichotomous outcome within 90 days following cardiac surgery. However, as supplementary analyses the number of readmissions as well as time to hospital readmission within 90 days will be included.

\section{Prolonged length of stay}

Prolonged length of stay will be measured by the number of days in the ICU, as well as total length of hospital stay. 
Table 2 Number of patients, expected number of outcomes within 90 days after cardiac surgery and included predictive variables and covariates for datasets $1,2,3$ and 4

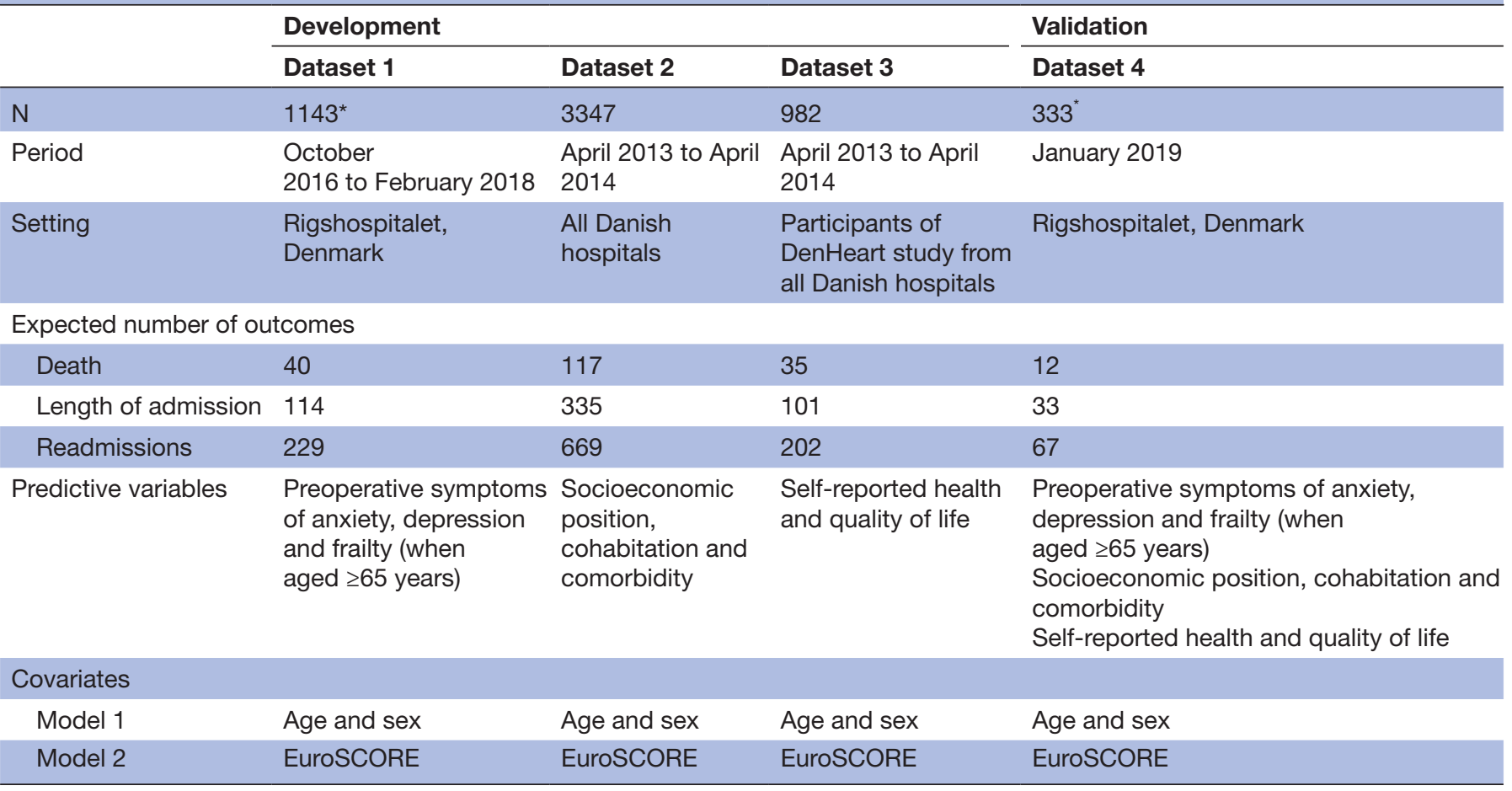

*Estimated number of patients to be included based on expected incidence of death following cardiac surgery of $3.5 \%$.

The length of stay outcome is used as a proxy for complications assuming minor complications will increase the length of the total hospital stay and major complications will increase the length of stay in the ICU.

Length of hospital stay, and ICU stay will be dichotomised to designate normal and prolonged length of stay.

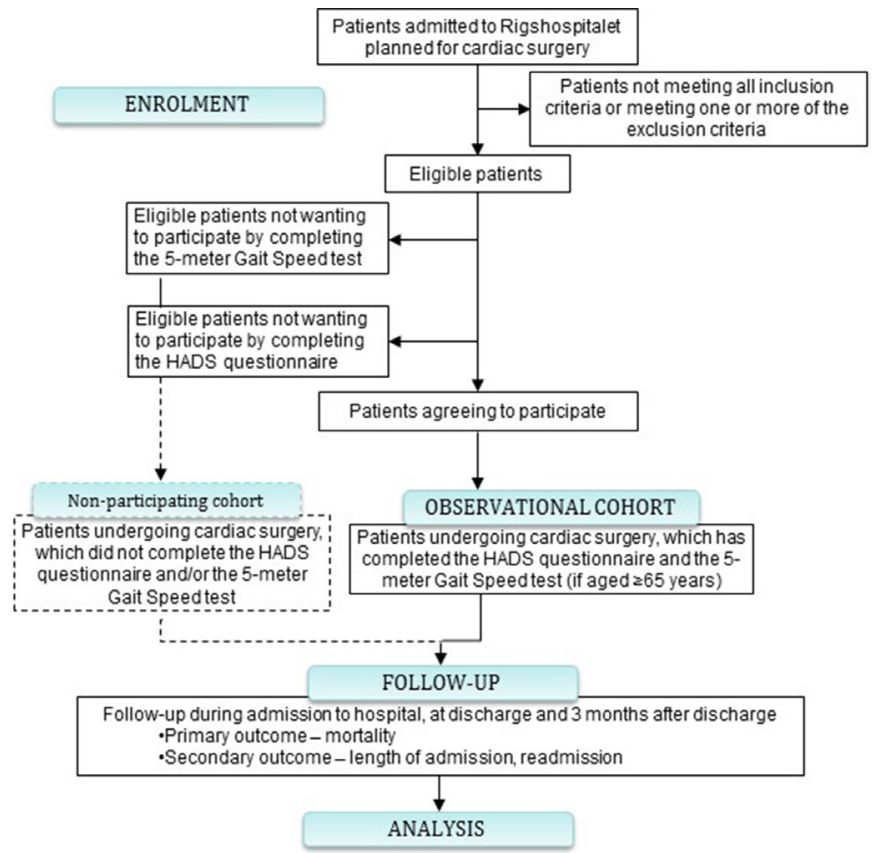

Figure 2 Flowchart, dataset 1. HADS, Hospital Anxiety and Depression Scale.
There is no consensus on the definition of prolonged length of stay following cardiac surgery. Previous studies have adopted the 75th percentile of the length of stay distribution, while others have defined prolonged length of stay as hospitalisation of $>11$ days following cardiac surgery, ${ }^{3}{ }^{44}$ which will be used in this study.

Duration of stay in the ICU is a common endpoint in cardiac surgical studies since it is a standard component of the treatment. It provides indication of the patient's recovery profile and is in effect a composite measure of the entire perioperative process. ${ }^{30}$ Prolonged ICU stay is rare, being required in $<10 \%$ of operated patients but it

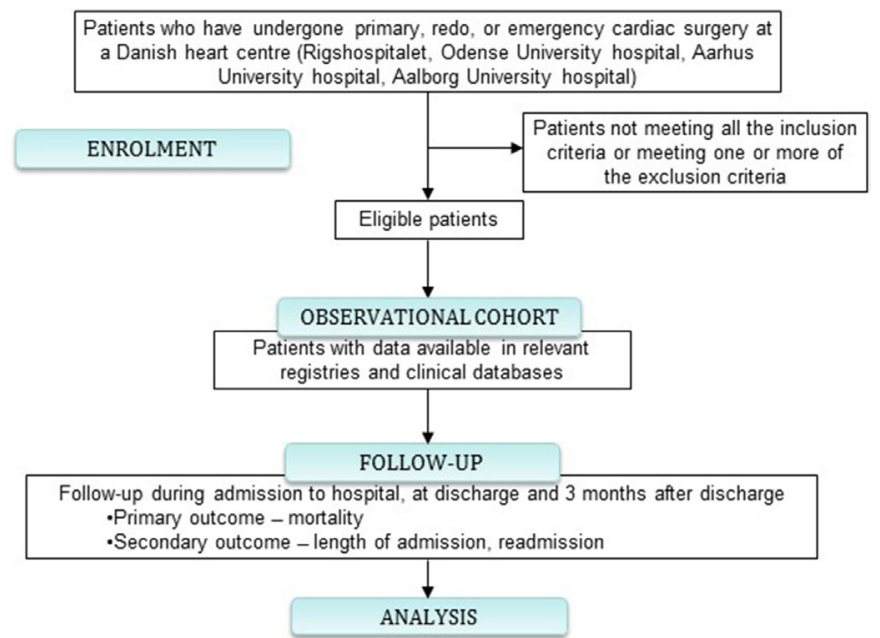

Figure 3 Flowchart, dataset 2. 


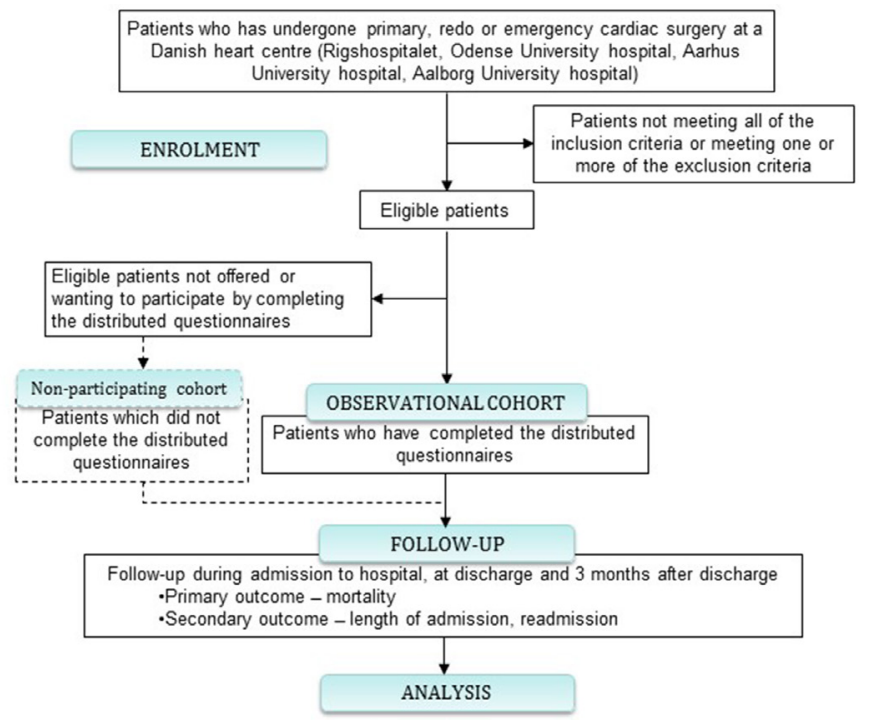

Figure 4 Flowchart, dataset 3.

utilises $>30 \%$ of all the ICU resources needed for cardiac surgery. ${ }^{45}$ In previous studies, prolonged length of stay in the ICU has been defined as $>24$ to $>96$ hours. $^{3}{ }^{46-51}$ For the present study, based on existing literature and clinical framework, prolonged length of stay in the ICU is defined as $>72$ hours. To further quantify the degree of complications leading to prolonged admission to the ICU, supplementary analyses will be performed for selected ICU-related outcomes such as mechanical ventilation and use of inotropes or vasopressors. ${ }^{52}$

\section{Predictors, rationale and measures}

With this study, variables reflecting emotional, behavioural, social and functional factors will be evaluated. All suggested predictors including categorisation are presented in table 1.

Potential predictors were chosen based on their clinical relevance, prior findings of an association with the outcomes $^{68}$ and the expected number of outcomes (tables 1 and2). The expected number of outcomes is listed in table 2.

\section{Emotional factors}

In patients undergoing cardiac surgery knowledge of prevalence and influence of emotional factors is lagging behind the evidence documented in other heart conditions. In cardiac surgery, the emphasis has been on preserving cognitive function rather than mental health per se. ${ }^{53}$ Included emotional factors are anxiety, depression and health-related quality of life (HRQoL). Patients undergoing cardiac surgery will be assessed for symptoms of anxiety and depression 1 day prior to surgery (dataset 1) with the Hospital Anxiety and Depression Scale (HADS), a validated questionnaire assessing depression and anxiety in hospitalised patients. ${ }^{54}$ A score of 7 or less indicates non-cases, scores of 8-10 for doubtful cases and scores of 11 or more for definite cases. ${ }^{54}$
HRQoL will be measured by HeartQoL and 12-Item Short-Form Health Survey (SF-12).

HeartQoL is a disease-specific tool comprising 14 items, with 10-item physical and 4-item emotional subscales, which are scored from 0 (poor HRQoL) to 3 (best). ${ }^{55}$ With Cronbach's $\alpha$ between 0.80 and 0.91 for the global score and each subscale, the tool has been found reliable in patients with a wide range of cardiac diagnoses. ${ }^{55}$ HeartQoL quantities are converted to binary quantities based on the median score.

The SF-12 constitutes the measure of mental and physical health. ${ }^{56}$ SF-12 is a generic measure shown useful in measuring physical and mental health even in smaller populations. ${ }^{57}{ }^{58}$ Individual mental component summary scores and physical component summary scores (PCS) ranging from 0 to 100 will be calculated based on the user's manual. ${ }^{58}$ Higher scores indicate better health states. As recommended the cut-off will be set as the mean minus one SD, using the Danish normed score. ${ }^{57}$

\section{Social factors}

Several social factors have been found to be associated with outcomes following cardiac surgery. Measures of socioeconomic status (SES) have been found to influence disease incidence, treatment and health outcomes. Those with lower income, lower education or working in lower status occupations experience increased mortality and morbidity. ${ }^{22} 252759$ SES will be measured by highest attained educational level and household equivalised disposable income, which is the household disposable income divided by the number of members of the household converted into equalised adults. For this study, the equivalised disposable income will be stratified by $\leq 50 \%$ median, $51 \%-149 \%$ median and $\geq 150 \%$ median. Highest attained educational level will be defined as a maximum of 10 years of school, high school, vocational, higher education, undergraduate or postgraduate. Information on socioeconomic factors will be obtained from registers at Statistics Denmark. Furthermore, living alone has been linked with poor health outcomes in earlier studies, which may reflect that patients who are socially isolated are more likely to smoke and drink alcohol heavily, ${ }^{60}{ }^{61}$ delay seeking treatment, ${ }^{62}$ demonstrate non-compliance with medical regimens ${ }^{63}$ and may be due to lack of emotional or practical support gained through living with another person. ${ }^{64}$ The complexity of the influence of social connections on disease morbidity and mortality is simplified in a model by Holt-Lunstad and Smith shown in figure $5 .{ }^{65}$ Both married and cohabiting couples are included, as $>20 \%$ of all couples living together in Denmark are in non-marital cohabiting relationships. To further investigate the influence of social connections, patients were asked two further questions in the DenHeart study-whether they experienced having someone to talk to if they needed support or were having problems and if they sometimes were alone, even though they would prefer to be with others. 


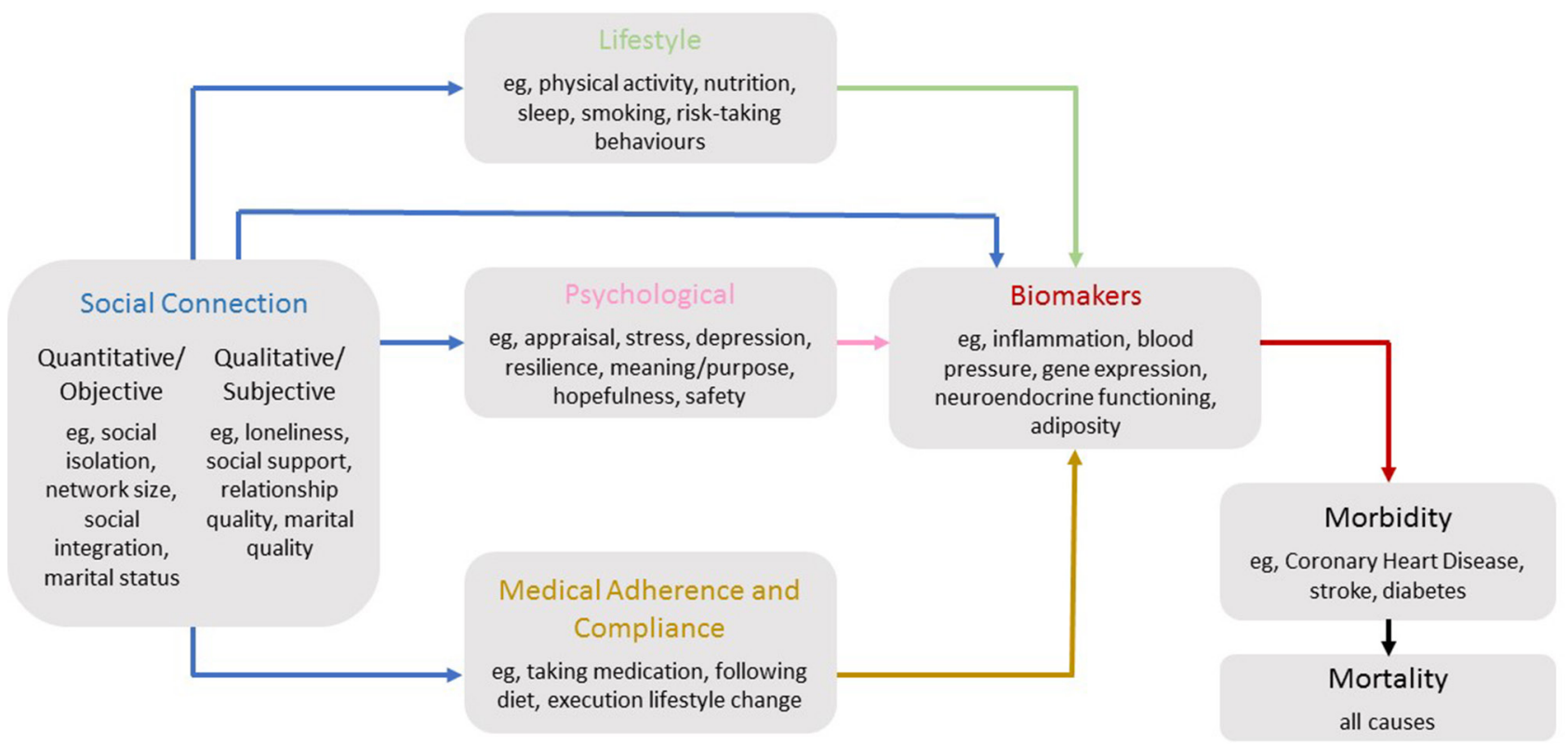

Figure 5 A simplified model of possible direct and indirect pathways by which social connections influence disease morbidity and mortality. ${ }^{66}$

\section{Functional factors}

Improved functional status is a major goal for most patients undergoing cardiac surgery. Indeed, disability, functional decline and frailty have been found to be predictive of mortality, major morbidity and complications following cardiac surgery ${ }^{66-69}$ For this study, frailty will be measured preoperatively in patients aged $70+$ years by the 5 -metre Gait speed test.

Emotional and cognitive functioning will be measured by the Brief Illness Perception Questionnaire (B-IPQ), which has eight items comprising cognitive and emotional illness perceptions. Each dimension is assessed on a scale from 0 to 10 with higher scores indicating stronger perceptions. Five of the items assess cognitive illness representations, two assess emotional representations and one item assesses illness comprehensibility. ${ }^{70} 71$ The eight items (consequences, timeline, personal control, treatment control, identity, illness concern, coherence and emotional representation) may be combined as one total score, or each item may be assessed separately. ${ }^{72}$ No clear cut-offs for screening have been determined for B-IPQ. To reduce the df only the summary score will be included for the main analyses in three categories based on the 25th and 75th quartile in the studied sample.

\section{Behavioural factors}

Behavioural factors have previously been linked with outcomes following cardiac surgery. Several studies demonstrate that health-risk behaviours such as smoking, alcohol consumption, sedentary lifestyle and obesity are associated with a variety of diseases and mortality ${ }^{73}$ Smoking status will be included as current, ex-smoker and never smoking. Heavy alcohol consumption will be defined as $>14 / 21 \mathrm{U} /$ week for women and men, respectively. BMI was considered for EuroSCORE II, but BMI was only weakly related to mortality risk $(\mathrm{p}=0.08)$ and was therefore not included in the final model. However, in the current study BMI will be included since outcomes other than mortality are included.

Complications related to anaesthesia and surgery are important to patients and expensive for the healthcare system. Postoperative complications result in increased morbidity and mortality, and extended hospital stay and convalescence.

\section{Statistical analysis}

Data management will proceed without examining predictor-outcome associations. Continuous predictors are categorised (table 2). In case of patients undergoing surgery more than once in the inclusion periods, only the first entry will be included.

All analyses will consist of separate models for each of the datasets 1, 2 and 3 since they represent different populations.

The questionnaires described above, HeartQoL (physical) and SF-12 (PCS) both measure a physical component of HRQoL. In order to be able to reduce the $\mathrm{df}$, a correlation analysis on the two scales in dataset 3 will be conducted. In case of high correlations, the scale with the strongest association with outcomes will be chosen for inclusion.

To evaluate selection bias in the included data, a non-responder analysis will be conducted to compare responders and non-responders in the survey data by register-based information (datasets 1 and 3).

In case of missingness of predictors, single mean imputation for each item will be performed for continuous variables while for categorical variables missing values will be assigned to the category most frequently occurring. If $>10 \%$ are missing, best-worst case analyses will be done 
as sensitivity analyses where best case means that patients with primary outcome will have low predictor level and worst case means that patients with primary outcome will have highest predictor level. This will be done to evaluate the most extreme possible datasets.

\section{Variable selection}

Logistic regression analyses will be used to investigate the relationship between the prognostic variables and the outcomes.

Since the interest of this study is to examine whether emotional, behavioural, social and functional factors add to the existing EuroSCORE, the incremental value of each added variable will be determined by comparison of the predictive value of the model with and without the added variables in addition to the EuroSCORE. As the first descriptive reporting, each candidate predictor variable will be included separately in a model with the EuroSCORE. In the model development, each potential predictive variable will be removed stepwise from the model by using an automated backwards selection procedure. We will use the Akaike's Information Criterium to determine which predictors to include and set a liberal significance level of 0.10 .

\section{Scoring}

The risk score will be calculated by a sum of the products of individual values of each predictor variable and its regression coefficient. ${ }^{74}$ To examine the performance of the prognostic model, patients will be classified as low $(<3 \%)$, moderate $(3 \%-5 \%)$ and high $(>5 \%)$ risk.

\section{Model performance}

To examine the apparent performance (internal validity) of the prognostic screening tools, we will assess the risk score on discrimination, calibration and overall performance.

Discrimination refers to the ability of the model to separate patients who develop events from those who do not. ${ }^{75}$ To identify the discrimination of the model only including EuroSCORE and the final model, c-statistics for discriminative ability will be estimated and the receiver operating characteristic curve (ROC) by area under the ROC curve (AUC) will be presented.

Calibration measures how accurately the model's predictions match overall observed event rates. ${ }^{75}$ We will evaluate the calibration of the model only including the EuroSCORE and the final model by a calibration plot, where we plot the observed outcome by decile of the predictions. ${ }^{76}$ To quantify the performance of the model in terms of calibration the calibration plot will be characterised by an intercept a, which indicates the extent that predictions are systematically too low or too high, and a calibration slope $\mathrm{b}$, which should be $1 .^{76}$

To measure the overall performance of the model, the scaled Brier score will be applied by calculating the distance between the predicted outcome and actual outcome taking account of the maximum Brier value.
The scaled Brier score will range from $0 \%$ with a perfect fitting model to $100 \%$ for a non-informative model. ${ }^{76}$

Adjustment for competing risks due to mortality will be included in the statistical analyses repeating the evaluation of discrimination, calibration and model performance among patients that survive at least 90 days after surgery.

Measures of discrimination, calibration and model performance may not be sensitive when including new predictors to an existing model. ${ }^{76}$ Therefore, when comparing the model only with the EuroSCORE with the final model, we will also calculate measures of reclassification. This is done by supplementary analyses evaluating how many patients change categories (low, medium and high risks as defined above) in the final model compared with the model only including EuroSCORE. $^{77}$

\section{Validation stage}

In this stage, we will complete a prospective study to determine the predictive validity of the derived risk assessment tool. For the external model validation predictor and outcome values will be measured among other patients from a validation cohort providing independent data. The derived model, with its predictors and assigned weights, as estimated from the development stage will be applied to these data, and the model's predictive performance will be quantified. ${ }^{78}$

\section{Validation sample (dataset 4)}

The validation sample will consist of a dataset including every adult patient undergoing primary, redo or emergency cardiac surgery at one large urban hospital. Included patients will be scored and classified according to the derived prediction model from the development stage as having low $(<3 \%)$, moderate $(3 \%-5 \%)$ or high $(>5 \%)$ risk of outcomes occurring. Patients will then be followed for mortality, prolonged length of admission and readmission up to 3 months after cardiac surgery in nationwide registers and clinical databases. For patients from the validation sample which have missing information on some of the predictors in the risk model mean imputation will be performed. The model will be evaluated by calculating the measures of discrimination, calibration and model performance as described above. Information on expected sample size and number of outcomes is available in table 2.

In case of poor performance of the final model in the validation sample, updating of the model will be performed by re-estimation of predictor weights or adding or removing existing predictors from the original model. $^{79}$

\section{Presentation of the model}

Based on the performance of the model in the validation sample, a nomogram or online calculator will be developed. 


\section{Patient and public involvement}

The described study does not include patient or public involvement since part of the data collection had been performed when the idea for the study was formulated. This is the first time patient reported outcomes are included in a risk assessment model for cardiac surgery, based on knowledge that several patient reported outcomes have been found to be associated with hard endpoints. Thus, it was not relevant for the current study to include patient preferences; however, findings from this study will be disseminated among patients and patient organisations.

\section{DISCUSSION}

This paper describes the material and statistical analysis plan to develop and validate a prognostic screening tool for patients undergoing cardiac surgery, following the PROGRESS framework and TRIPOD reporting guidelines. The prognostic screening tool consists of the existing EuroSCORE with added variables reflecting emotional, behavioural, social and functional factors. Several of the included predictors are based on existing data measured in previously collected datasets; however, some predictors were based on data primary collected for this study. The analysis of existing data is an expedient way to make full use of data that are already collected to address potentially important new research questions, ${ }^{80}$ and furthermore an effort to avoid disturbing patients unnecessarily. The study described uses corresponding datasets from multiple studies. When doing this, there is a risk that the datasets differ in important aspects, such as baseline risk. However, in the described study a prediction model will be developed for each dataset avoiding bias due to this. In the external validation stage, the models will be combined and tested in one dataset. ${ }^{78}$

The risk score calculations available for datasets 2 and 3 are calculated based on EuroSCORE I and not the updated EuroSCORE II. EuroSCORE I has been found to over-estimate ${ }^{12}$ and EuroSCORE II to under-estimate mortality. ${ }^{138182}$ It is therefore reasonable to assume that the potential improvement of the model is not attributable to the choice of EuroSCORE model.

Potential predictors chosen for this study have in earlier studies been found to be associated with poor outcomes following cardiac surgery, not only from Denmark, but internationally. Furthermore, potential predictors included in this study represent common traits and issues that should be easily collected in different settings. Thus, results from this study has the potential to be generalised to countries outside of Denmark. However, external validation on datasets from other institutions are required to confirm this.

According to the TRIPOD guidelines in prediction model studies, the outcome is ideally assessed while blinded to information about the predictors. ${ }^{33}$ The predictors may otherwise influence the outcome assessment. However, this risk is limited for the objective outcomes included in the present study where the collection of outcome data is from administrative registers without the influence of the investigator. Furthermore, the quality of the follow-up data is exceptional for Denmark due to the comprehensive national registers. ${ }^{83}$

The relative importance of calibration and discrimination ultimately depends on the purpose of the screening tool. Thus, if the purpose of the tool is to aid clinical decision-making and provides accurate estimates of risk to patients, then calibration is most important. If clinicians were to inform patients of their risk of dying following cardiac surgery, this estimate would be misleading if the tool was not well calibrated and, for example, more patients with the same level of risk than estimated died following cardiac surgery. If, on the other hand, the purpose of the tool is to select appropriate patients to include in a randomised trial, for example, in a trial with the purpose to reduce poor outcome following cardiac surgery then adequate discrimination is important. In that case, a poorly discriminating tool would misclassify a large proportion of patients, including several inappropriate (low risk) patients and excluding appropriate (high risk) patients.

The suggested study of the development and validation of a prognostic screening tool for patients undergoing cardiac surgery may provide a foundation to determine the need for clinical interventions aimed at reducing the risk associated with cardiac surgery. Furthermore, the addition of non-medical parameters can potentially improve the risk evaluation process to make the prediction by risk assessment models more accurate and with higher discrimination leading to greater public acceptance of the resulting decisions.

\section{CONCLUSION}

This protocol outlines the design of the developing and validating studies for a prognostic screening tool for patients undergoing cardiac surgery. Results coming from this study will be interpreted for both clinical and research purposes.

\section{ETHICS AND DISSEMINATION}

The study will follow the requirements from the Ethical Committee System ensuring voluntary participation in accordance with the Helsinki declarations. According to Danish legislation, surveys do not have to be approved by an ethics committee system. A consent form was signed by all participating patients.

\footnotetext{
Author affiliations

${ }^{1}$ Department of Cardiothoracic Anaesthesiology, Rigshospitalet, Copenhagen, Denmark

${ }^{2}$ Heart Centre, Rigshospitalet, Copenhagen, Denmark

${ }^{3}$ The National Institute of Public Health, University of Southern Denmark, Copenhagen, Denmark

${ }^{4}$ Department of Public Health and Primary Care, KU Leuven, Leuven, Belgium
} 
${ }^{5}$ Institute of Health and Care Sciences, University of Gothenborg, Gothenborg, Sweden

${ }^{6}$ Department of Cardiothoracic Surgery, Rigshospitalet, Copenhagen, Denmark ${ }^{7}$ Department of Cardiothoracic Surgery, Papworth Hospital NHS Foundation Trust, Cambridge, UK

Contributors PFC and SKB conceived the idea for the study. PFC, SKB, LCT and PM initiated the study design and execution of the study. PFC and LCT described the statistical analysis plan. PFC wrote the first draft of the manuscript. SN revised the manuscript with a special focus on its relation to the original EuroSCORE model. SD assisted with knowledge of cardiac surgery both for the planning and designing of the study and when revising the manuscript. All authors revised the manuscript critically. All authors have given their final approval of the version to be published.

Funding This work was supported by Helsefonden grant number (16-B-0205), Hjertefonden, grant number (18-R124-A8454-22099) and Fabrikant Karl G. Andersens Fond, grant number (9292).

Competing interests None declared.

Patient consent for publication Not required.

Ethics approval The study was approved by the Danish Data Protection Agency (RH-2016-158) and the Danish Patient Safety Authority (3-3013-1930/1).

Provenance and peer review Not commissioned; externally peer reviewed.

Open access This is an open access article distributed in accordance with the Creative Commons Attribution Non Commercial (CC BY-NC 4.0) license, which permits others to distribute, remix, adapt, build upon this work non-commercially, and license their derivative works on different terms, provided the original work is properly cited, appropriate credit is given, any changes made indicated, and the use is non-commercial. See: http://creativecommons.org/licenses/by-nc/4.0/.

\section{REFERENCES}

1. Ngaage DL, Griffin S, Guvendik L, et al. Changing operative characteristics of patients undergoing operations for coronary artery disease: impact on early outcomes. Ann Thorac Surg 2008;86:1424-30.

2. Ferguson TB, Hammill BG, Peterson ED, et al. A Decade of Change - Risk Profiles and Outcomes for Isolated Coronary Artery Bypass Grafting Procedures, 1990 - 1999 : A Report From the STS National Database Committee and the Duke. Ann Thorac Surgery 2002;4975:1990-9.

3. Almashrafi A, Alsabti $\mathrm{H}$, Mukaddirov $\mathrm{M}$, et al. Factors associated with prolonged length of stay following cardiac surgery in a major referral hospital in Oman: a retrospective observational study. BMJ Open 2016;6:e010764.

4. Iribarne A, Chang $\mathrm{H}$, Alexander $\mathrm{JH}$, et al. Readmissions after cardiac surgery: experience of the National Institutes of Health/Canadian Institutes of Health research cardiothoracic surgical trials network. Ann Thorac Surg 2014;98:1274-80.

5. Gordon RS. An operational classification of disease prevention. Public Health Rep 1983;98:107-9.

6. Hemingway $\mathrm{H}$, Croft $\mathrm{P}$, Perel $\mathrm{P}$, et al. Prognosis research strategy (PROGRESS) 1: a framework for researching clinical outcomes. BMJ 2013;346:e5595.

7. Hingorani AD, Windt DA, Riley RD, et al. Prognosis research strategy (PROGRESS) 4: stratified medicine research. BMJ 2013;346:e5793-9.

8. Cromhout PF, Moons P, Thygesen LC, et al. Time to expand risk evaluation systems for cardiac surgery? Looking beyond physiological parameters. Eur J Cardiovasc Nurs 2018;17:760-6.

9. Nashef SA, Roques F, Michel P, et al. European system for cardiac operative risk evaluation (EuroSCORE). Eur J Cardiothorac Surg 1999;16:9-13.

10. Nashef SA, Roques F, Sharples LD, et al. EuroSCORE II. Eur J Cardiothorac Surg 2012;41:734-45.

11. Roques F, Nashef SA, Michel P, et al. Risk factors and outcome in European cardiac surgery: analysis of the EuroSCORE multinational database of 19030 patients. Eur J Cardiothorac Surg 1999;15:816-23.

12. Gogbashian A, Sedrakyan A, Treasure T. EuroSCORE: a systematic review of international performance. Eur J Cardiothorac Surg 2004;25:695-700.

13. Garcia-Valentin A, Mestres CA, Bernabeu E, et al. Validation and quality measurements for EuroSCORE and EuroSCORE II in the
Spanish cardiac surgical population: a prospective, multicentre study. Eur J Cardiothorac Surg 2016;49:399-405.

14. Borracci RA, Rubio M, Celano L, et al. Prospective validation of EuroSCORE II in patients undergoing cardiac surgery in Argentinean centres. Interact Cardiovasc Thorac Surg 2014;18:539-43.

15. Zhang GX, Wang $C$, Wang $L$, et al. Validation of EuroSCORE II in Chinese patients undergoing heart valve surgery. Heart Lung Circ 2013;22:606-11.

16. Celano CM, Huffman JC. Depression and cardiac disease: a review. Cardiol Rev 2011;19:130-42.

17. Mallik S, Krumholz HM, Lin ZQ, et al. Patients with depressive symptoms have lower health status benefits after coronary artery bypass surgery. Circulation 2005;111:271-7.

18. Stenman M, Holzmann MJ, Sartipy U. Relation of major depression to survival after coronary artery bypass grafting. Am J Cardiol 2014;114:698-703.

19. Terada T, Johnson JA, Norris C, et al. Severe obesity is associated with increased risk of early complications and extended length of stay following coronary artery bypass grafting surgery. J Am Heart Assoc 2016;5:9-11.

20. Ji Q, Zhao H, Mei Y, et al. Impact of smoking on early clinical outcomes in patients undergoing coronary artery bypass grafting surgery. J Cardiothorac Surg 2015;10:16.

21. Maheshwari A, Dalton JE, Yared JP, et al. The association between alcohol consumption and morbidity and mortality in patients undergoing coronary artery bypass surgery. J Cardiothorac Vasc Anesth 2010;24:580-5.

22. Koch CG, Li L, Kaplan GA, et al. Socioeconomic position, not race, is linked to death after cardiac surgery. Circ Cardiovasc Qual Outcomes 2010;3:267-76

23. Kaplan GA, Keil JE, Factors S. and Cardiovascular disease : a review of the literature. Circulation 1993;88:1973-98.

24. Tang KL, Rashid R, Godley J, et al. Association between subjective social status and cardiovascular disease and cardiovascular risk factors: a systematic review and meta-analysis. BMJ Open 2016;6:e010137.

25. Holme I, Helgeland A, Hjermann I, et al. Coronary risk factors and socioeconomic status. The Oslo study. Lancet 1976;2:1396-8.

26. Smith GD, Hart C, Watt G, et al. Individual social class, area-based deprivation, cardiovascular disease risk factors, and mortality: the Renfrew and Paisley Study. J Epidemiol Community Health 1998;52:399-405.

27. Alter DA, Naylor CD, Austin P, et al. Effects of socioeconomic status on access to invasive cardiac procedures and on mortality after acute myocardial infarction. N Engl J Med 1999;341:1359-67.

28. Afilalo J, Eisenberg MJ, Morin JF, et al. Gait speed as an incremental predictor of mortality and major morbidity in elderly patients undergoing cardiac surgery. J Am Coll Cardiol 2010;56:1668-76.

29. Sepehri A, Beggs T, Hassan A, et al. The impact of frailty on outcomes after cardiac surgery: a systematic review. J Thorac Cardiovasc Surg 2014;148:3110-7.

30. Myles PS. Meaningful outcome measures in cardiac surgery. J Extra Corpor Technol 2014;46:23-7.

31. Steyerberg EW, Moons KG, van der Windt DA, et al. Prognosis Research Strategy (PROGRESS) 3: prognostic model research. PLoS Med 2013;10:e1001381

32. Riley RD, Hayden JA, Steyerberg EW, et al. Prognosis Research Strategy (PROGRESS) 2: prognostic factor research. PLoS Med 2013;10:e1001380.

33. Moons KG, Altman DG, Reitsma JB, et al. Transparent Reporting of a multivariable prediction model for Individual Prognosis or Diagnosis (TRIPOD): explanation and elaboration. Ann Intern Med 2015;162:W1-73.

34. Collins GS, Reitsma JB, Altman DG, et al. Transparent Reporting of a Multivariable Prediction Model for Individual Prognosis or Diagnosis (TRIPOD): The TRIPOD Statement. Eur Urol 2015;67:1142-51.

35. Baumgartner $\mathrm{H}$, Falk V, Bax JJ, et al. ESC/EACTS Guidelines for the management of valvular heart disease. Eur Heart $J$ 2017;2017:2739-91.

36. Kolh P, Windecker S, Alfonso F, et al. ESC/EACTS Guidelines on myocardial revascularization. Eur J Cardio-Thoracic Surg 2014;2014:517-92.

37. Siregar S, Groenwold RH, de Mol BA, et al. Evaluation of cardiac surgery mortality rates: 30-day mortality or longer follow-up? Eur J Cardiothorac Surg 2013;44:875-83.

38. Berg SK, Svanholm J, Lauberg A, et al. Patient-reported outcomes at hospital discharge from Heart Centres, a national cross-sectional survey with a register-based follow-up: the DenHeart study protocol. BMJ Open 2014;4:e004709.

39. Lynge E, Sandegaard JL, Rebolj M. The Danish national patient register. Scand J Public Health 2011;39:30-3. 
40. Pedersen CB. The Danish civil registration system. Scand J Public Health 2011;39:22-5.

41. Schmidt M, Schmidt SA, Sandegaard JL, et al. The Danish National Patient Registry: a review of content, data quality, and research potential. Clin Epidemiol 2015;7:449

42. VestDansk Hjertedatabase. Yearly report 2016. 2017.

43. Christensen Al, Ekholm O, Glümer C, et al. The Danish National Health Survey 2010. Study design and respondent characteristics. Scand J Public Health 2012;40:391-7.

44. Papachristofi O, Klein AA, Mackay J, et al. Effect of individual patient risk, centre, surgeon and anaesthetist on length of stay in hospital after cardiac surgery: Association of Cardiothoracic Anaesthesia and Critical Care (ACTACC) consecutive cases series study of 10 UK specialist centres. BMJ Open 2017;7:e016947.

45. Pölönen $P$, Hippeläinen $M$, Takala R, et al. Relationship between intra- and postoperative oxygen transport and prolonged intensive care after cardiac surgery: a prospective study. Acta Anaesthesiol Scand 1997:41:810-7.

46. Abrahamyan L, Demirchyan A, Thompson ME, et al. Determinants of morbidity and intensive care unit stay after coronary surgery. Asian Cardiovasc Thorac Ann 2006;14:114-8.

47. Azarfarin R, Ashouri N, Totonchi Z, et al. Factors influencing prolonged ICU stay after open heart surgery. Res Cardiovasc Med 2014;3:e20159.

48. Nakasuji M, Matsushita M, Asada A. Risk factors for prolonged ICU stay in patients following coronary artery bypass grafting with a long duration of cardiopulmonary bypass. J Anesth 2005;19:118-23.

49. Atoui $R, M a F$, Langlois $Y$, et al. Risk factors for prolonged stay in the intensive care unit and on the ward after cardiac surgery. $J$ Card Surg 2008;23:99-106.

50. Ettema RG, Peelen LM, Schuurmans MJ, et al. Prediction models for prolonged intensive care unit stay after cardiac surgery: systematic review and validation study. Circulation 2010;122.

51. Janssen DP, Noyez L, Wouters C, et al. Preoperative prediction of prolonged stay in the intensive care unit for coronary bypass surgery. Eur J Cardiothorac Surg 2004;25:203-7.

52. Christiansen CF, Møller MH, Nielsen $\mathrm{H}$, et al. The Danish intensive care database. Clin Epidemiol 2016;8:525-30.

53. Tully PJ. Psychological depression and cardiac surgery: a comprehensive review. J Extra Corpor Technol 2012;44:224-32.

54. Zigmond AS, Snaith RP. The hospital anxiety and depression scale. Acta Psychiatr Scand 1983:67:361-70.

55. Oldridge N, Höfer S, McGee H, et al. The HeartQoL: Part I. Development of a new core health-related quality of life questionnaire for patients with ischemic heart disease. Eur J Prev Cardiol 2014;21:90-7.

56. Ware J, Kosinski M, Keller SD. A 12-Item Short-Form Health Survey: construction of scales and preliminary tests of reliability and validity. Med Care 1996;34:220-33.

57. Gandek B, Ware JE, Aaronson NK, et al. Cross-validation of item selection and scoring for the SF-12 Health Survey in nine countries: results from the IQOLA Project. International Quality of Life Assessment. J Clin Epidemiol 1998;51:1171-8.

58. Ware JE, Kosinski M, Turner-Bowker DM, et al. User's manual for the SF-12v2 health survey, 2007.

59. Smith GD, Hart C, Blane D, et al. Lifetime socioeconomic position and mortality: prospective observational study. BMJ 1997;314:547-52.

60. Dickens CM, McGowan L, Percival C, et al. Lack of a close confidant, but not depression, predicts further cardiac events after myocardial infarction. Heart 2004;90:518-22.

61. Haustein KO. Smoking and poverty. Eur J Cardiovasc Prev Rehabil 2006;13:312-8.

62. Schwarz B, Schoberberger R, Rieder A, et al. Factors delaying treatment of acute myocardial infarction. Eur Heart $J$ 1994;15:1595-8.
63. DiMatteo MR, Lepper HS, Croghan TW. Depression is a risk factor for noncompliance with medical treatment: meta-analysis of the effects of anxiety and depression on patient adherence. Arch Intern Med 2000;160:2101-7.

64. Murphy BM, Elliott PC, Le Grande MR, et al. Living alone predicts 30-day hospital readmission after coronary artery bypass graft surgery. Eur J Cardiovasc Prev Rehabil 2008;15:210-5.

65. Holt-Lunstad J, Smith TB. Loneliness and social isolation as risk factors for CVD: implications for evidence-based patient care and scientific inquiry. Heart 2016;102:987-9.

66. Afilalo J, Mottillo S, Eisenberg MJ, et al. Addition of frailty and disability to cardiac surgery risk scores identifies elderly patients at high risk of mortality or major morbidity. Circ Cardiovasc Qual Outcomes 2012;5:222-8.

67. Feuchtinger J, Halfens R, Dassen T. Pressure ulcer risk assessment immediately after cardiac surgery--does it make a difference? A comparison of three pressure ulcer risk assessment instruments within a cardiac surgery population. Nurs Crit Care 2007;12:42-9.

68. van der Mast RC, van den Broek WW, Fekkes D, et al. Incidence of and preoperative predictors for delirium after cardiac surgery. $J$ Psychosom Res 1999;46:479-83.

69. Blumenthal JA, Lett HS, Babyak MA, et al. Depression as a risk factor for mortality after coronary artery bypass surgery. Lancet 2003;362:604-9.

70. Broadbent E, Petrie KJ, Main J, et al. The brief illness perception questionnaire. J Psychosom Res 2006;60:631-7.

71. Broadbent $\mathrm{E}$, Wilkes $\mathrm{C}$, Koschwanez $\mathrm{H}$, et al. A systematic review and meta-analysis of the Brief Illness Perception Questionnaire. Psychol Health 2015;30:1361-85.

72. Igwesi-Chidobe CN, Coker B, Onwasigwe CN, et al. Biopsychosocial factors associated with chronic low back pain disability in rural Nigeria: a population-based cross-sectional study. BMJ Glob Health 2017;2:e000284.

73. Institute of Medicine (US) Committee on Health and Behavior: Research Practice and Policy. Health and behavior : the interplay of biological, behavioral, and societal influences. Washington DC: National Academy Press, 2001.

74. Adams ST, Leveson SH. Clinical prediction rules. BMJ 2012;344:d8312.

75. Pencina MJ, D'Agostino RB. Evaluating discrimination of risk prediction models: the C Statistic. JAMA 2015;314:1063-4.

76. Steyerberg EW, Vickers AJ, Cook NR, et al. Assessing the performance of prediction models: a framework for traditional and novel measures. Epidemiology 2010;21:128-38.

77. Cook NR. Use and misuse of the receiver operating characteristic curve in risk prediction. Circulation 2007;115:928-35.

78. Debray TP, Moons KG, Ahmed I, et al. A framework for developing, implementing, and evaluating clinical prediction models in an individual participant data meta-analysis. Stat Med 2013;32:3158-80.

79. Moons KG, Kengne AP, Grobbee DE, et al. Risk prediction models: II. External validation, model updating, and impact assessment. Heart 2012;98:691-8.

80. Cheng HG, Phillips MR. Secondary analysis of existing data: opportunities and implementation. Shanghai Arch Psychiatry 2014;26:371.

81. Kalender M, Adademir T, Tasar M, et al. Validation of EuroSCORE II risk model for coronary artery bypass surgery in high-risk patients. Kardiochir Torakochirurgia Pol 2014;11:252-6.

82. Noyez L, Kievit PC, van Swieten HA, et al. Cardiac operative risk evaluation: The EuroSCORE II, does it make a real difference? Neth Heart J 2012;20:494-8.

83. Thygesen LC, Daasnes C, Thaulow I, et al. Introduction to Danish (nationwide) registers on health and social issues: structure, access, legislation, and archiving. Scand $J$ Public Health 2011;39:12-16. 